\title{
1858 : une année faste pour les sociétés immobilières en ville de Neuchâtel
}

\section{Claire Piguet}

\section{(2) OpenEdition}

1 Journals

Édition électronique

URL : http://journals.openedition.org/edl/237

DOI : 10.4000/edl.237

ISSN : 2296-5084

Éditeur

Université de Lausanne

\section{Édition imprimée}

Date de publication : 15 décembre 2010

Pagination : 155-180

ISBN : 978-2-940331-24-6

ISSN : 0014-2026

\section{Référence électronique}

Claire Piguet, « 1858 : une année faste pour les sociétés immobilières en ville de Neuchâtel », Études de lettres [En ligne], 4 | 2010, mis en ligne le 15 décembre 2013, consulté le 22 décembre 2020. URL:

http://journals.openedition.org/edl/237 ; DOI : https://doi.org/10.4000/edl.237 


\section{8: UNE ANNÉE FASTE POUR LES SOCIÉTÉS IMMOBILIÈRES EN VILLE DE NEUCHÂTEL}

Dans un contexte de profonds changements économiques, politiques et sociaux, cinq sociétés immobilières voient le jour à Neuchâtel entre mai 1858 et juillet 1859 . Reflet de leurs tailles et de leurs objectifs, elles offrent un nombre et un éventail de réalisations très variés: d'une à quarante constructions dans le domaine du logement et des infrastructures. Si la figure de l'architecte est discrète, la forte implication des autorités municipales constitue par contre une surprise.

\section{Cinq d'un coup}

C'est à Neuchâtel que maintenant on bâtit le plus. Trois sociétés de construction y sont en pleine activité, la Société de construction, la société Maret et Ritter et la société immobilière. La première élève actuellement deux quartiers de maisons, l'un entre la place Pury et le lac, l'autre dans les vignes du Tertre, achetées de la famille de Rougemont [...]. La seconde société continue à repeupler le quartier de VieuxChâtel. La troisième, qui s'est constituée dans le but de construire des habitations saines et à bon marché pour la classe ouvrière, bâtit un quartier de maisons simples, bien distribuées et bien exposées, entre l'Ecluse et les Parcs. A côté de ces trois sociétés, une quatrième édifie près du gymnase un vaste hôtel pour les voyageurs, l'hôtel Bellevue ${ }^{1}$.

Une cinquième «s'occupe de l'érection d'un manège près du Crêt » ${ }^{2}$ La création en quelques mois de cinq sociétés de construction en ville de

I. Le véritable messager boiteux de Neuchâtel, 1860, p. 41.

2. Le véritable messager boiteux de Neuchâtel, 1859, p. 41 sq. 
Neuchâtel constitue un événement assez marquant pour figurer dans la revue Le véritable messager boiteux de Neuchâtel. Plutôt que d'approcher chronologiquement la question du développement des sociétés immobilières au XIX ${ }^{\mathrm{e}}$ siècle, arrêtons-nous sur ce moment prolixe et tentons de cerner les paramètres dont relève ce phénomène ${ }^{3}$.

A défaut de disposer des archives de ces cinq établissements, les traces laissées dans les fonds publics et dans le tissu urbain permettent de dresser une première image, notamment celle de leurs débuts et de leurs liens avec les autorités. Il faut par contre abandonner toute velléité d'analyse de leur gestion financière, de leur rendement, de leur fonctionnement ou des mouvements de leur actionnariat.

\section{Une nouvelle organisation administrative}

Chef-lieu du canton éponyme depuis 1848, Neuchâtel demeure une petite ville à l'échelle de la Suisse et de l'Europe, même si sa population atteint 10000 habitants au début des années 1860 et double durant le troisième quart du XIX ${ }^{\mathrm{e}}$ siècle ${ }^{4}$.

A cette époque, les autorités cantonales et communales sortent d'une période de crise suscitée par le changement de régime de 1848, suivi de la tentative avortée de restauration royaliste de 1856 . Conséquence de ces bouleversements, la gestion politique et administrative de la ville prend un tour nouveau en 1856, date à laquelle la Municipalité de Neuchâtel apparaît comme seconde administration. Jusqualors seuls les bourgeois étaient représentés au sein de la Commune et jouissaient de droits politiques. L'établissement d'un système municipal permet désormais à l'ensemble des habitants de la commune de jouir de ces prérogatives. Ces réformes politiques et administratives secouent toutefois sérieusement les habitudes et les mentalités. La répartition des compétences, des services publics et des revenus entre les deux administrations ne se règle

3. Cette étude s'inscrit dans la prolongation de premières recherches publiées dans les articles suivants: C. Piguet, "Mécanismes urbains et pièces d'architecture»; Id., "Arrêt sur la ville»; Id., "Panorama de la construction de logements à bon marché dans le canton de Neuchâtel (1850-1914)».

4. 7821 habitants en 1851, 9521 en 1860 et 13557 en 1875. Archives de la ville de Neuchâtel (abrégé: AVN), Rapport de comptabilité et de gestion pour l'exercice [de l'année] présenté au Conseil général par le Conseil municipal (abrégé: rapport de gestion + année). 
pas sans mal et nécessite l'intervention du Conseil d'Etat, du Grand Conseil et finalement du Tribunal fédéral pour parvenir à une convention en 1861. La Commune bourgeoise défend le plus longtemps possible ses attributions dans les domaines de l'assistance et de l'éducation du niveau secondaire, ainsi que dans la gestion de ses bâtiments, alors que la Municipalité reprend immédiatement la conduite des dicastères de l'instruction primaire et des travaux publics. Sur décision du Conseil d'Etat, la Commune bourgeoise est finalement dissoute en 1888, le solde de ses services revenant à la Municipalité qui devient alors l'unique entité politique sous le vocable de la Commune.

\section{Un moment charnière dans le développement de la ville de Neuchâtel}

Freinée dans ses velléités d'extension par une topographie en pente et ponctuée de "crêts", ainsi que par l'absence de surface plane naturelle, Neuchâtel s'affranchit toutefois de son cadre médiéval avec la création de faubourgs dès le XVIII' siècle 5 .

De 1839 à 1864, le détournement du Seyon permet l'assainissement et la transformation du noyau ancien; à la traditionnelle traversée routière est-ouest s'ajoute un axe nord-sud qui revêt rapidement une fonction commerciale, alors qu'une place est aménagée dans l'ancien delta de la rivière. Remarquons également les timides tentatives de comblements des rives du lac, la modeste extension du tissu bâti, la rareté des infrastructures et des bâtiments publics, ainsi que la concentration de l'industrie dans le vallon de la Serrière.

En 1860, Neuchâtel termine la restructuration précoce de son centre historique et se trouve à l'aube d'un processus de croissance sans précédent, la cité de l'ancien régime cédant progressivement la place à une petite ville industrielle et commerciale.

Malgré son souci de prévenir un développement anarchique de la localité, de ménager la possibilité de travaux d'édilité et d'encourager les mesures d'hygiène et de prévention contre le feu, la Municipalité ne se dote d'aucune véritable politique en matière d'urbanisme avant la fin du

5. Pour un panorama complet du développement de Neuchâtel durant la seconde moitié du XIX ${ }^{\mathrm{e}}$ siècle, voir J. Courvoisier, O. Girardbille, J.-P. Jelmini et Ch. Rodeschini, «Neuchâtel, histoire d'un paysage urbain» et C. Piguet, «Neuchâtel». 
XIX siècle. Au contraire des villes de La Chaux-de-Fonds et du Locle, elle ne dispose pas d'outils de contrôle et d'urbanisation comme un plan d'extension et son règlement ${ }^{6}$. La Direction des Travaux publics, sa Commission des bâtiments, ainsi que la Police du feu sanctionnent les alignements des rues et les constructions au coup par coup, sans concept ou vision d'ensemble, l'essor urbain s'apparentant davantage à une croissance organique qu'à un développement rationnel.

\section{Les sociétés immobilières...}

"Ce qui a donné un certain essor à l'industrie du bâtiment dans le canton, c'est la formation des sociétés de construction. ${ }^{7}$ Feuilleter les manuels du Conseil d'Etat ou les registres d'enregistrement des traités d'entreprise corrobore le constat établi a posteriori par l'ingénieur Gustave de Pury. Loin de se cantonner au seul domaine de l'immobilier, les sociétés et les associations en tous genres se sont en effet multipliées au cours des années 1850 dans le canton de Neuchâtel, notamment dans les secteurs industriels, culturels ou philanthropiques ${ }^{8}$. De la société simple à la société anonyme, leur statut juridique revêt également un large éventail de formes:

Les unes, organisées à peu près comme les maisons de commerce ordinaires composées de quelques associés opérant sous leurs noms, ont construit des bâtiments dont le nombre et l'importance étaient nécessairement limités par les mises de fonds et le crédit des intéressés.

6. Les villes des Montagnes neuchâteloises disposent de plans établis durant les années 1830 et révisés entre 1854 et 1859 . En 1834-1835, Neuchâtel est doté d'un plan d'alignement qui n'a pas servi au-delà de la restructuration du centre. Voir C. Piguet, «Neuchâtel », p. 166 sq.; Id., "Mécanismes urbains et pièces d'architecture», p. 182-185.

7. G. de Pury, «L'activité neuchâteloise dans le domaine de la construction pendant les trente dernières années", p. 263.

8. Citons notamment la Société des bâtiments du Cercle des Bayards (1855), l'Association immobilière du Locle (1855), la Société de construction de La Chaux-de-Fonds (1855), la Société du Manège de La Chaux-de-Fonds (1855), la Société de construction de La Chaux-du-Milieu (1856), la Société du Cercle démocratique de Travers (1858), la Société du musée Challandes (1858), l'Association ouvrière au Locle (1858), la Société de la Salle des Concerts de Neuchâtel (1860) qui apparaissent dans les tables des Manuels du Conseil d'Etat de 1853 à 1860. 
Les autres, constituées sous forme de sociétés anonymes avec émission d'actions et souvent d'obligations, disposent en général de ressources plus considérables puisque, pour plusieurs d'entre elles, le fonds capital s'élève à fr. $1000000^{9}$.

Dans le domaine de la construction, onze sociétés anonymes sont ainsi sanctionnées par le Grand Conseil en une vingtaine d'années de 1853 à $1879^{10}$, sans compter les fréquentes associations de professionnels du bâtiment qui se forment à l'occasion d'un chantier ou d'un projet particulier.

Sans surprise, de telles créations répondent à des aspirations financières (faire fructifier l'argent de particuliers ou de collectivités), à des motivations pratiques (rassembler suffisamment de capitaux pour permettre la réalisation de projets d'envergure) et à des besoins d'utilité publique (créer des logements ou des infrastructures).

Les modifications légales intervenues au milieu du XIX ${ }^{\mathrm{e}}$ siècle se révèlent favorables aux sociétés axées sur le domaine de la construction. En 1852 , la législation neuchâteloise révise sa loi de $1833^{11}$ pour la mettre en accord avec la Loi fédérale sur les sociétés anonymes. De leur côté, les lods (droits de mutation sur toute vente d'immeuble) passent de $10 \%$ à $4 \%$ en $1852^{12}$, juste avant qu'ils ne soient supprimés pour le transfert d'actions de certaines sociétés anonymes ayant pour objet la construction d'immeubles en 1856 et $1857^{13}$.

Cinq sociétés immobilières ont été créées en ville de Neuchâtel en l'espace de quelques mois - de mai 1858 à juillet 1859. L'examen de leurs statuts à l'aide d'une fiche descriptive type ${ }^{14}$ a permis de démêler points communs et spécificités. Leur objectif principal consiste à édifier

9. G. de Pury, «L'activité neuchâteloise dans le domaine de la construction pendant les trente dernières années", p. 263.

Io. Ibid., p. 263 sq.

II. Décret concernant les sociétés anonymes du 10 décembre 1852. La création d'une société anonyme est notamment soumise à la sanction du Grand Conseil.

I2. Loi sur la réduction des lods du 3 décembre 1851.

I3. Décret exemptant du paiement des lods le transfert des actions de certaines Sociétés anonymes, du 25 novembre 1856 et décret régularisant, quant aux lods, la position de certaines Sociétés anonymes fondées dans le but de construire des bâtiments, du 2 octobre 1857.

I4. Les rubriques sont les suivantes: raison sociale, date (enregistrement), durée, siège social, fonds social, rendement proposé, buts, composition de l'actionnariat, 
des bâtiments, mais leur nombre peut varier d'une réalisation unique à quelque trente-cinq à quarante constructions. La localisation de leur siège social, ainsi que leur rayon d'action, correspondent au territoire communal. En général prévues pour une durée de vingt à trente ans, certaines sociétés respectent strictement le délai, alors que d'autres sont dissoutes avant terme ou prolongées ${ }^{15}$. A l'exception de la Société de Vieux-Châtel qui est en commandite, les quatre autres sont des sociétés anonymes qui tombent sous l'égide de la loi fédérale du même nom et possèdent une structure similaire. L'Assemblée générale réunit les actionnaires et définit le mandat du Conseil d'administration qui est parfois secondé d'un Comité de direction. Du dédommagement symbolique aux rétributions fixes, le traitement de l'exécutif est très variable et tributaire de la taille et des objectifs de la société. L'actionnariat se voit en général proposer un rendement de $5 \%$, à l'exception des initiatives à vocation philanthropique ou d'utilité publique ${ }^{16}$, sans qu'il ne nous ait été possible de vérifier les résultats financiers et les dividendes effectivement touchés. Quant au fonds social, il reflète la vocation et les ambitions de la société. Il débute à 25000 francs dans le cas du manège, monte à 100000 francs pour la Société immobilière pour la classe ouvrière, puis à 300000 francs pour les projets de Vieux-Châtel et de l'hôtel Bellevue, avant d'atteindre finalement 1000000 francs, en ce qui concerne la Société de construction.

\section{5. ... et leurs réalisations}

\subsection{Hôtel Bellevue}

A l'initiative d'Albert Elskes, «maître d'hôtel» et «fondateur de l'entreprise», secondé par cinq personnalités neuchâteloises, la Société de l'Hôtel Bellevue est créée en juillet 1859. Elle «a pour objet la construction d'un hôtel à Neuchâtel, près de l'embarcadère des bateaux à vapeur.

organisation (organe), composition du Conseil d'administration, modification ou renouvellement, dissolution, réalisations, résultats financiers, réception.

I5. La Société de Vieux-Châtel fait exception avec sa durée d'existence de six ans.

16. Un dividende de $3 \%$ est prévu pour les actionnaires de la Société immobilière pour la classe ouvrière, alors que rien n'est prévu pour ceux de la Société du manège. 
Cet hôtel une fois construit sera meublé aux frais de la Société \& remis en location aux conditions qui seront fixées par l'Assemblée Générale» ${ }^{17}$. Son principal actionnaire avait préalablement pris une option sur le terrain situé à l'ouest du port ${ }^{18}$; la parcelle est finalement vendue à la nouvelle société contre une prise d'actions équivalente à sa valeur numéraire, les autorités espérant ainsi faire un placement intéressant, tout en soutenant le développement du tourisme ${ }^{19}$. Un mandat est ensuite confié à l'architecte Hans Rychner pour l'élaboration des plans et la réalisation de l'édifice (fig. 1).

Jouissant d'une excellente implantation à proximité du port, l'hôtel, réalisé de 1859 à 1861, dispose de trois façades offrant la vue sur le lac et les Alpes (fig. 2) ${ }^{20}$. Le bâtiment répond aux canons de l'architecture néoclassique (volume cubique, toiture plate, ordonnance tripartite des façades, vocabulaire ornemental, etc.). Seule dérogation aux habitudes constructives régionales, la couverture d'asphalte ne résiste malheureusement pas au climat et doit être convertie en un toit à quatre pans en 1876.

A l'intérieur, les quatre-vingts chambres des étages, ainsi que les salles à manger, salon, fumoir et bureau du rez-de-chaussée, sont distribuées autour d'un puits de lumière central cerné de galeries de circulation. Avec les bains et les communs situés au sous-sol, l'hôtel dispose d'un équipement à la pointe du confort.

Créée pour réunir les capitaux nécessaires à l'édification et au lancement de l'hôtel Bellevue, la société est dissoute en $1876^{21}$, lorsqu'Albert Elskes, à la fois locataire et actionnaire, devient l'unique propriétaire

I7. Archives de l'Etat de Neuchâtel (abrégé: AEN), Archives judiciaires, Neuchâtel, dos. 168, traité des sociétés, "Société de l'Hôtel de Belle-Vue», sanctionné par le Grand-Conseil le 28 juin 1859 et déposé au Greffe du Tribunal civil le 5 juillet 1859. Augmentation du fonds social de 300000 à 350000 francs: "Société de l'Hôtel de Bellevue à Neuchâtel, appendice à l'acte constitutif de la Société de l'Hôtel de Belle-Vue à Neuchâtel en date du 27 mai 1859, du 29 juillet 1861 ».

I8. AVN, procès-verbaux (abrégé: PV) du Conseil général de la Municipalité, 14 décembre 1858.

19. AVN, PV du Conseil municipal, 5 et 12 janvier, 23 février, 4, 9 et 23 mars, 22 juillet et 21 septembre 1859. AEN, manuel du Conseil d'Etat (abrégé: MCE), 6 septembre 1859 .

20. Rue du Musée 1. C. Piguet, "Neuchâtel», p. 246.

2I. AEN, Archives judiciaires, Neuchâtel, dos. 169, traité des sociétés, "Société du Grand Hôtel Bellevue, du 14 juillet 1876, dissolution de société». 

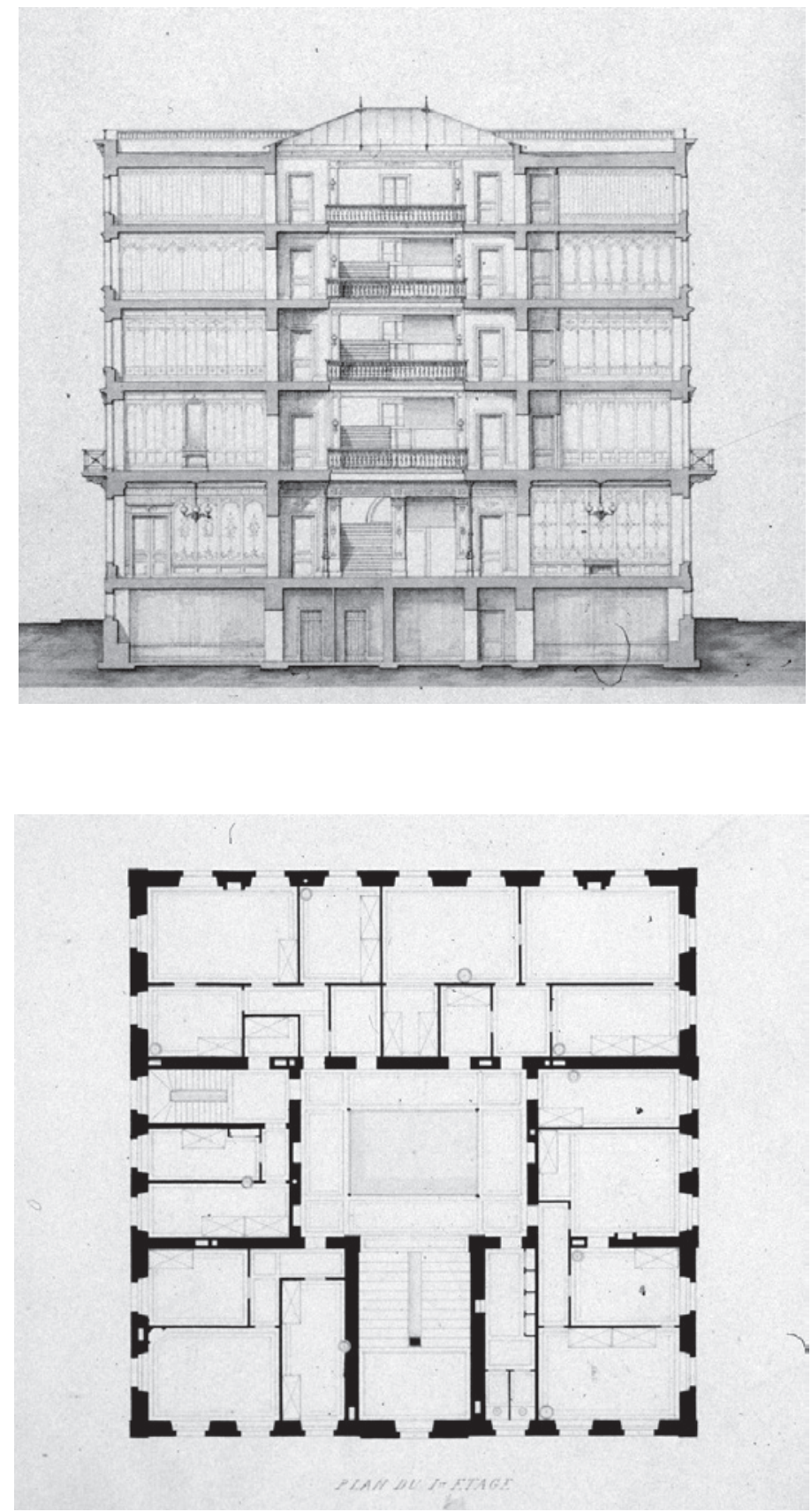

Fig. 1 - Hôtel Bellevue, coupe et plan par Hans Rychner, 1859. (AVN). 


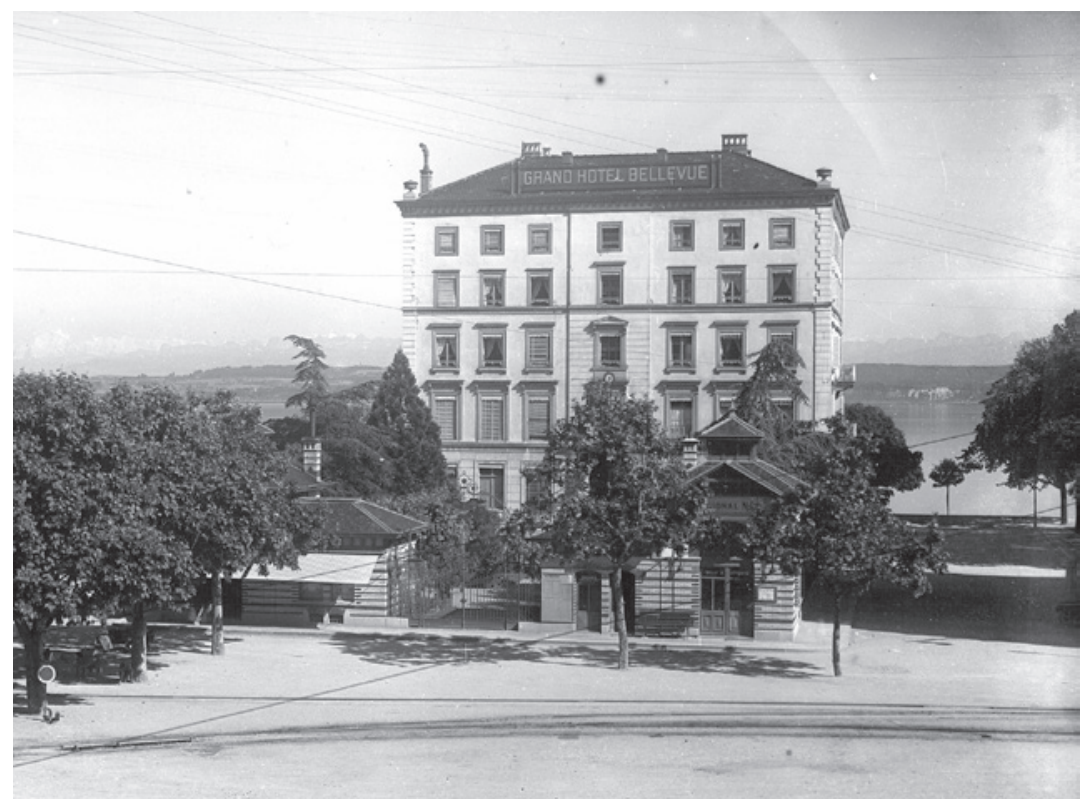

Fig. 2 - Hôtel Bellevue, vue générale de l'édifice avec sa nouvelle toiture, vers 1900. (Institut suisse pour la conservation de la photographie, collection V. Attinger).

de l'hôtel, conformément à l'une des clauses statutaires lui permettant de racheter progressivement les actions émises. L'établissement cessera ses activités pour être vendu et reconverti en bâtiment administratif en 1920.

\subsection{Manège}

Trente et un gradés de l'armée et amateurs d'équitation lancent en 1858 une pétition pour obtenir un terrain à l'Ecluse sur lequel édifier le manège qui manque alors à l'équipement du chef-lieu; «le bâtiment terminé, il sera loué à un entrepreneur ou administré en régie par la société» ${ }^{22}$. Le Conseil général refuse le projet, mais accepte le principe

22. AEN, Archives judiciaires, Neuchâtel, dos. 167, traité des sociétés, «Société du Manège de Neuchâtel», sanctionné par le Grand-Conseil le 29 juillet 1858 et déposé au Greffe du Tribunal civil de Neuchâtel le 17 août 1858. Augmentation du capital de 25000 à 35000 francs, en raison de la nécessité d'acheter le terrain à la Commune bourgeoise. AEN, MCE, 7 juin 1859 et approbation du Grand-Conseil, le 21 juin 1859. 
d'une "cession de terrain gratuite ou [d'une] allocation en écus» ${ }^{23}$. Après avoir rallié un total de septante-neuf membres fondateurs à leur cause, le comité parvient à obtenir une subvention municipale de 1000 francs en raison du "caractère éminemment utile» de cette initiative et surtout du soutien concurrent de la Commune bourgeoise qui offre 5500 francs $^{24}$.

Détenteur d'une unique action, l'architecte Hans Rychner se voit confier la réalisation en 1858-1859 d'un bâtiment à l'architecture plus utilitaire que prestigieuse et qui n'a par conséquent guère laissé de trace iconographique $^{25}$.

Assurant les travaux d'entretien et la mise en location, la Société poursuit son activité durant plus de soixante ans, son existence étant prolongée en 1881 et en $1908^{26}$, avant que le manège ne soit vendu à la Commune en 1917 et démoli en 1929.

\subsection{Société de Vieux-Châtel}

Déjà associés au sein de la Société Maret Ritter et $\mathrm{Cie}^{27}$, le notaire CharlesHenri Maret, le professeur Edouard Desor et l'ingénieur Guillaume Ritter forment une nouvelle société ayant pour but «la construction de bâtiments sur le terrain acheté au quartier de Vieux-Châtel» à la famille Coulon ${ }^{28}$. Les directeurs de la Société Maret Ritter et Cie conjuguent ainsi leurs expertises commerciale, juridique, géologique, technique et constructive à un important apport de capitaux, de façon à insuffler une dimension nouvelle aux affaires de l'ancienne entreprise Ritter, active dans le domaine de la fourniture de matériaux, et dans l'espoir de développer le secteur architectural. La Municipalité refuse de participer à la construction

23. AVN, PV du Conseil général de la Municipalité, 14 juin, 21 octobre et 2 novembre 1858 .

24. AVN, PV du Conseil municipal, 16 juin, 17, 20, 25 et 27 août, 24 septembre, $1^{\text {er }}$, 8 et 25 octobre, 12 novembre 1858 et 7 janvier 1859 .

25. Rue de la Maladière 2. C. Piguet, «Neuchâtel», p. 243.

26. AEN, Archives judiciaires, Neuchâtel, dos. 169, traité des sociétés, "Société du Manège de Neuchâtel, du 28 juillet 1881, acte de prolongation de Société». La société du Manège est dissoute le 26 juin 1917 et radiée le 7 novembre 1927, selon les données du Registre du commerce de Neuchâtel.

27. Ladite société a été créée en 1857 (cf. C. Piguet, "Arrêt sur la ville», p. 53).

28. Rue de Vieux-Châtel 11-23. C. Piguet, "Neuchâtel», p. 269. AEN, fonds Guillaume Ritter, dos. 108, traité [de la Société de Vieux-Châtel], 12 juillet 1858. 


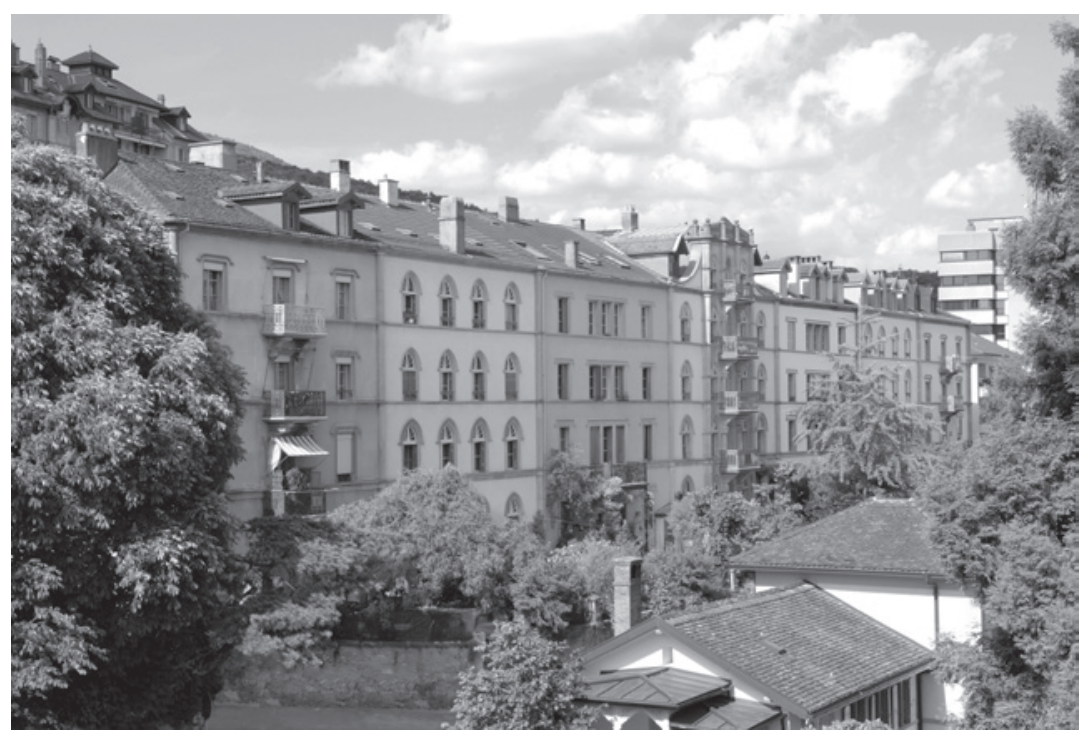

Fig. 3 - Vue générale du massif de Vieux-Châtel.

d'immeubles locatifs à Vieux-Châtel en qualité d'associé commanditaire, mais accepte de prêter une somme de 150000 francs.

Cette initiative aboutit à la réalisation en 1859-1860 d'un massif de sept immeubles locatifs contigus, complété en 1861 par des bûchers et des dépendances au nord (fig. 3). Imposantes pour l'époque en ville de Neuchâtel, les dimensions de cet ensemble (84,5 x 11,5 m), ainsi que l'implantation dans la pente ne sont pas sans rappeler la division tripartite (bâti, jardin, rue) qui caractérise l'urbanisme chaux-de-fonnier.

Vue de l'extérieur, la symétrie du massif est renforcée par l'accent mis sur le corps central, alors que les décors et la modénature d'inspiration néogothique sont très diversifiés (fig. 4). A l'intérieur, les matériaux sont traditionnels, mais la qualité de l'équipement (parquets, boiseries, ferronneries, persiennes, etc.) est remarquable ${ }^{29}$.

Résidence d'horlogers, de rentiers, de professeurs et de leurs domestiques ${ }^{30}$, l'ensemble propose des appartements spacieux et confortables

29. J.-B. Cotelli, «Les immeubles de Vieux-Châtel, un événement unique».

30. Liste des premiers habitants du massif de Vieux-Châtel établie par Adi-Pierre Glanzmann et publiée en annexe dans N. Bauermeister (dir.), Utopierre, Guillaume Ritter, de Vieux-Châtel à Neuchâtel, p. 181-184. 


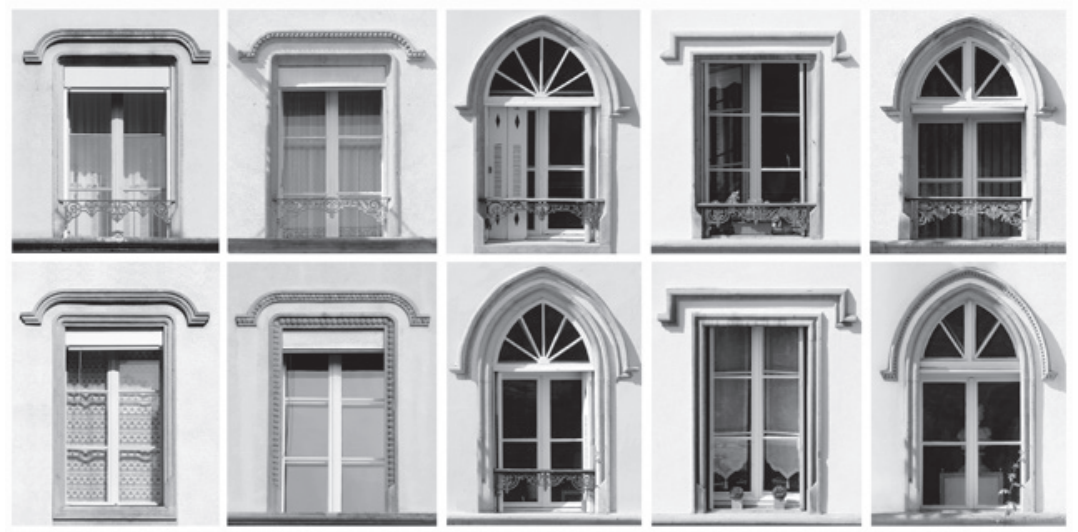

Fig. 4 - Eventail des encadrements de fenêtres.

qui comprennent trois chambres au sud, deux locaux au nord, sans compter le vestibule et le réduit qui donnent sur la cage d'escalier. Chacun des immeubles ne compte qu'un seul logement par étage. Directeur de la Société des eaux, Guillaume Ritter amènera les derniers perfectionnements en matière d'approvisionnement en eau: une fontaine en 1861, suivie du raccordement au système de distribution de la ville en $1867-1868^{31}$.

La Société de Vieux-Châtel disparaîtra une fois son objectif atteint, mais la réalisation servira de carte de visite à la Société de matériaux Maret Ritter et Cie, en offrant un catalogue de ses prestations ${ }^{32}$. Malgré les efforts déployés, les constructions seront moins nombreuses qu'espérées, mais la fourniture de matériaux contribuera à la fortune de ses propriétaires.

\subsection{Société immobilière pour la classe ouvrière}

Créée dans un esprit philanthropique, la Société immobilière pour la classe ouvrière a pour objet «la construction de maisons à Neuchâtel, destinées aux classes ouvrières et pauvres " ${ }^{33}$, des habitations qui sont

3I. C. Piguet, "Guillaume Ritter: un arroseur arrosé?».

32. J.-B. Cotelli, «Les immeubles de Vieux-Châtel, un événement unique», p. 81.

33. AEN, Archives judiciaires, Neuchâtel, dos. 168, traité des sociétés, "Société immobilière pour la classe ouvrière», 7 décembre 1858 et sanction du Grand-Conseil le 
vouées à la location, à la location-vente ou à la vente aux enchères. Douze notables locaux, vraisemblablement suivis d'autres actionnaires, acceptent ainsi un rendement de 3\% au lieu des $5 \%$ habituellement proposés. Avec l'aide de professionnels du bâtiment, les architectes Louis et Léo Châtelain probablement, le Conseil d'administration réalise les constructions et gère leur entretien. Une austérité qui sied à la classe ouvrière selon les critères de l'époque préside aux choix architecturaux (fig. 5). En 1860 déjà, la nouvelle cité de l'Immobilière compte vingthuit cellules familiales réparties en seize bâtiments mélangeant pavillons et massifs ${ }^{34}$. Le premier type comprend un groupe de quatre logis de deux étages chacun, directement inspiré de la célèbre cité ouvrière de Mulhouse ${ }^{35}$, alors que le second type est constitué d'une barre de trois maisonnettes accolées, inscrite dans la pente et offrant un logement à chacun des trois étages.

Selon un bilan tiré dix ans après sa conception, la réalisation présente dans un premier temps «trop d'analogie avec les cités ouvrières des grands centres industriels; [...] les habitants se trouvent trop séparés et trop isolés du reste de la population " ${ }^{36}$. Considérés comme éloignés du centre au moment de leur édification, les logements de la petite cité sont par contre très recherchés quarante ans plus tard parce qu'ils «ne sont plus à l'extrémité de la ville et que les bénéfices sont employés en très grande partie pour améliorer et assainir les logements. Ils possèdent l'eau sur l'évier, ils sont plus spacieux que la majorité des logements de la classe ouvrière, ils ont un petit jardin et beaucoup de soleil» ${ }^{37}$. Toujours en activité une génération plus tard, la Société immobilière se lance dans la

15 décembre 1858. C. Piguet, «Panorama de la construction de logements à bon marché dans le canton de Neuchâtel (1850-1914)», p. 15 sq. Devenue Société immobilière 1858 au milieu du XXe siècle, elle est toujours active en 1966, selon les données du Registre du commerce de Neuchâtel.

34. Ruelle de l'Immobilière 2-20 (vendu en 1982) et rue des Parcs 19 et 21 (démoli en 1982). C. Piguet, "Neuchâtel», p. 238.

35. Edifiée entre 1854 et 1890 pour la manufacture Dolfuss, la cité ouvrière de Mulhouse compte au final 1243 maisons abritant pour la plupart quatre logements. Conçue par l'architecte Emile Muller, elle constituera une référence durant de nombreuses années.

36. L. Châtelain, «Rapport sur les habitations à bon marché», p. 116.

37. E. Quartier-la-Tente, Le canton de Neuchâtel, le district de Neuchâtel, revue historique et monographique des communes du canton, le district de Neuchâtel, p. 485. 


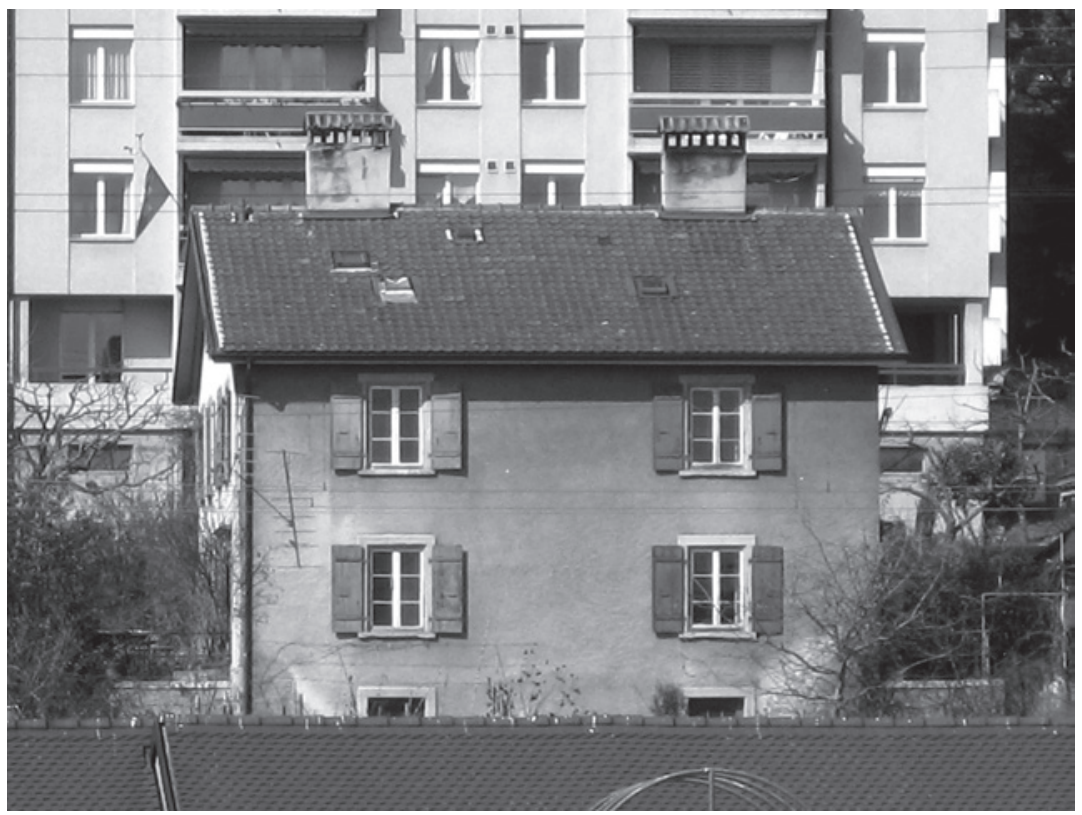

Fig. 5 - Détail d'une des maisonnettes de la cité de l'Immobilière. Etat en 2009.

construction d'un nouvel ensemble de trois maisons doubles en $1898^{38}$. Plus coûteuses que prévu, les maisons n'ont finalement pas permis aux familles ouvrières d'accéder à la propriété ${ }^{39}$.

\subsection{Société de construction}

Au milieu du XIX ${ }^{\mathrm{e}}$ siècle, Neuchâtel souffre d'une cruelle pénurie de logements. Dans l'espoir d'attirer l'industrie, notamment horlogère, la Municipalité décide de susciter la création d'une société de construction ${ }^{40}$ :

Il faut en premier lieu présenter au Conseil général un rapport avec projet d'arrêté sur la matière, et élaborer un projet de convention qui

38. Rue des Parcs 89-91, 93-95 et 97-99. C. Piguet, "Neuchâtel», p. 248.

39. E. Quartier-la-Tente, Le canton de Neuchâtel, le district de Neuchâtel, revue historique et monographique des communes du canton, le district de Neuchâtel p. 485.

40. AVN, PV du Conseil municipal, 27 avril 1857. 
détermine dans quelle mesure la Municipalité participera à la dite Société [de construction]; dès que ces préliminaires seront réglés, convoquer les personnes sur lesquelles on croit pouvoir compter pour cet établissement, et soumettre le projet de convention à leur délibération; la convocation une fois adoptée par les deux parties la société se trouverait par le fait constituée ${ }^{41}$.

Si «la construction de maisons dans la circonscription de Neuchâtel» est son but, la Société de construction fonctionne également comme une entreprise générale:

Pour réaliser ce but, la société acquerra les sols de maison et, s'il y a lieu, les carrières, forêts, etc. qui lui seront nécessaires; elle fera les plans et devis des constructions à élever, ainsi que les approvisionnements de matériaux dont elle aura besoin ; elle construira soit en régie, soit par voie d'adjudication ${ }^{42}$.

Ses statuts lui permettent également de vendre du sol à bâtir à des particuliers ou de leur fournir des constructions.

En dehors des initiateurs du projet, la société compte près de deux cent cinquante membres fondateurs, essentiellement neuchâtelois. Un actionnariat aussi fragmenté, de même qu'un volume de construction aussi ambitieux, demandent une structure bien outillée. La Société de construction dispose d'un état-major plus étoffé et professionnel que d'autres sociétés, son Comité directeur comprenant notamment l'ingénieur Gustave de Pury et l'architecte Louis Châtelain.

Sans véritable concurrence dans le chef-lieu, la Société de construction domine le marché de la construction durant ses vingt années d'existence, même si son ardeur est tempérée par les difficultés qui opposent la Municipalité et la Commune bourgeoise à propos des terrains, ainsi que par les réserves émises par le Grand Conseil pour empêcher la spéculation foncière qui «enlèverait à la libre construction des emplacements susceptibles de recevoir des bâtiments " ${ }^{43}$. Parmi ses nombreuses

4I. AVN, PV du Conseil municipal, 19 octobre 1857.

42. E. Bauer, Autour d'une place, p. 54 sq., ainsi que p. 54-62.

43. AEN, Bulletin officiel des délibérations du Grand Conseil de la République et canton de Neuchâtel, publié par ordre de l'Assemblée, vol. 18, Neuchâtel, 1857 [sic], p. 628. Toute augmentation du fonds social ou modification des statuts devra être soumise au Grand Conseil, alors que l'acquisition des terrains devra recevoir l'approbation du Conseil d'Etat et son sol utilisé dans un délai de dix-huit mois. 


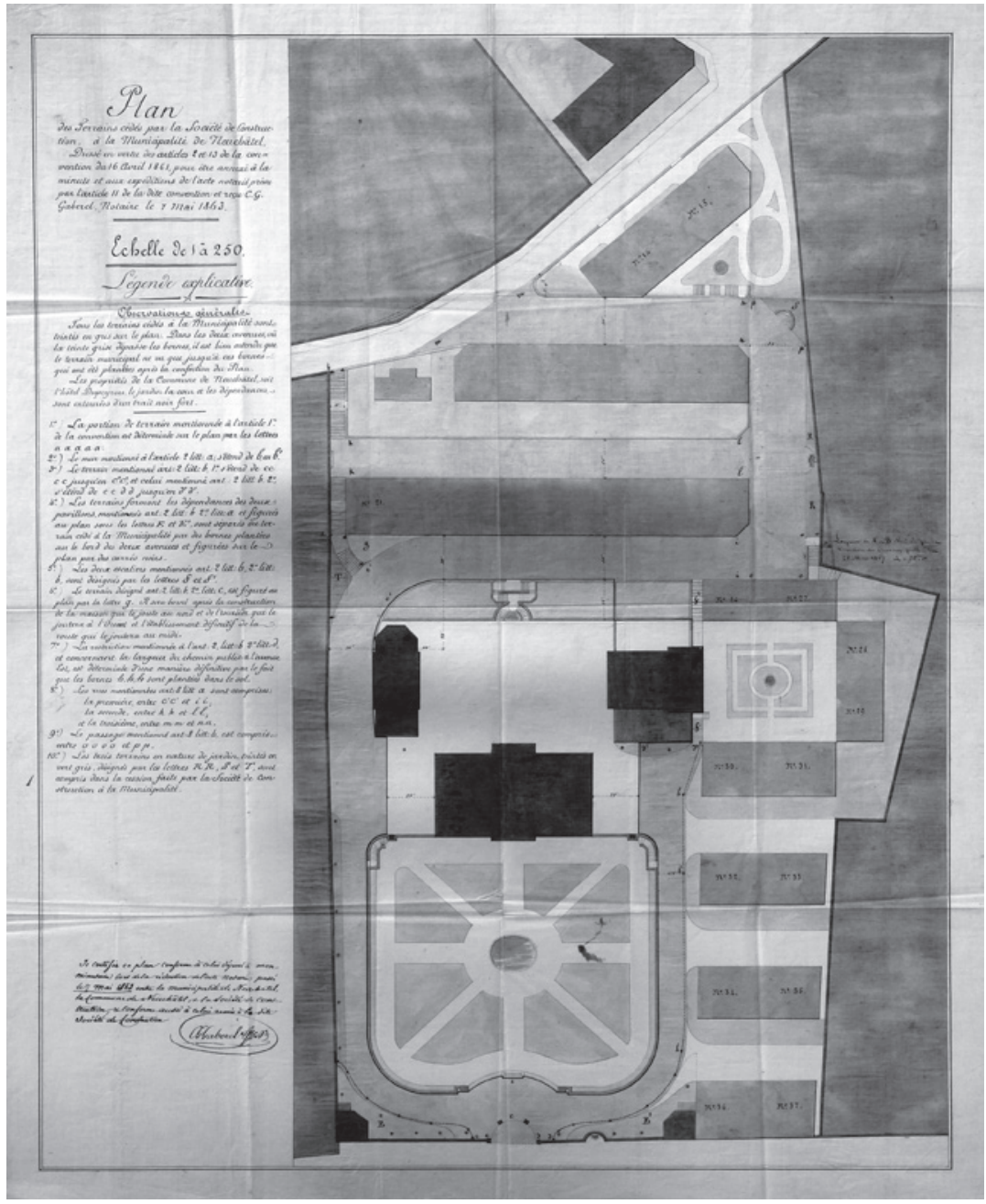

Fig. 6 - Plan de distribution d'une partie de la propriété DuPeyrou, 1863. (AVN). 
réalisations immobilières, deux projets majeurs marqueront l'urbanisme neuchâtelois: les lotissements de la propriété DuPeyrou (1859-1869) et du massif sud de la place Pury (1859-1865) ${ }^{44}$.

Vendue à la Société de construction en 1858 par les héritiers d'Albert de Rougemont, l'ancienne propriété DuPeyrou est rapidement démantelée, sur la base d'un plan établi par Louis Châtelain et englobant l'ancien hôtel particulier parmi les nouvelles constructions (fig. 6). La Société de construction se lance alors dans le percement des rues et l'édification des premiers massifs de bâtiments, le reste de la propriété faisant l'objet d'un morcellement en lots à bâtir sous son égide. La proximité de la ville et de la gare ainsi que l'exposition favorable destinent les alentours de l'hôtel DuPeyrou à l'édification d'immeubles résidentiels urbains, contrairement au quartier du Tertre, zone d'habitat populaire dans un repli naturel du terrain, et à la rue de l'Orangerie au bénéfice d'un emplacement plat, mais peu ensoleillé. Edifiés dans un style très urbain et cosmopolite, les immeubles comportent plusieurs logements dont la distribution, la grandeur et le confort varient en fonction de leur standing, un statut qui se reflète sur la richesse décorative des façades.

Etablies en 1844 par Achille-François-René Leclère, l'un des inspecteurs généraux des monuments de France, les dispositions générales de la place Pury sont mises en ouvre par l'architecte communal Henri Dietrich dès 1845 et achevées par son successeur Louis Châtelain. Dans une mise en scène urbaine inspirée de la place de la Concorde à Paris, la place de forme trapézoïdale est fermée au sud par deux massifs aux façades monumentales (fig. 7). Alors que le projet initial prévoyait la vente par lots et la construction des immeubles selon un cahier des charges assez strict, la révolution de 1848, le mauvais état des finances publiques, les difficultés du comblement et les nouvelles priorités en matière d'urbanisme provoquent des retards. Malgré l'édification d'un premier immeuble en 1853, le projet s'enlise, jusqu'à ce que la Société de construction accepte de se charger du chantier, moyennant quelques concessions au cahier des charges. Les constructions reprennent et le quartier est achevé en 1865. Le caractère résidentiel, parfois monumental et luxueux, vaudra à la Société de construction des critiques sur la nature élitaire de ses constructions. Avec l'homogénéité de ses façades en pierre

44. C. Piguet, «Neuchâtel», p. 180, 199, 228, 232, 245-247, 258 et 261. 


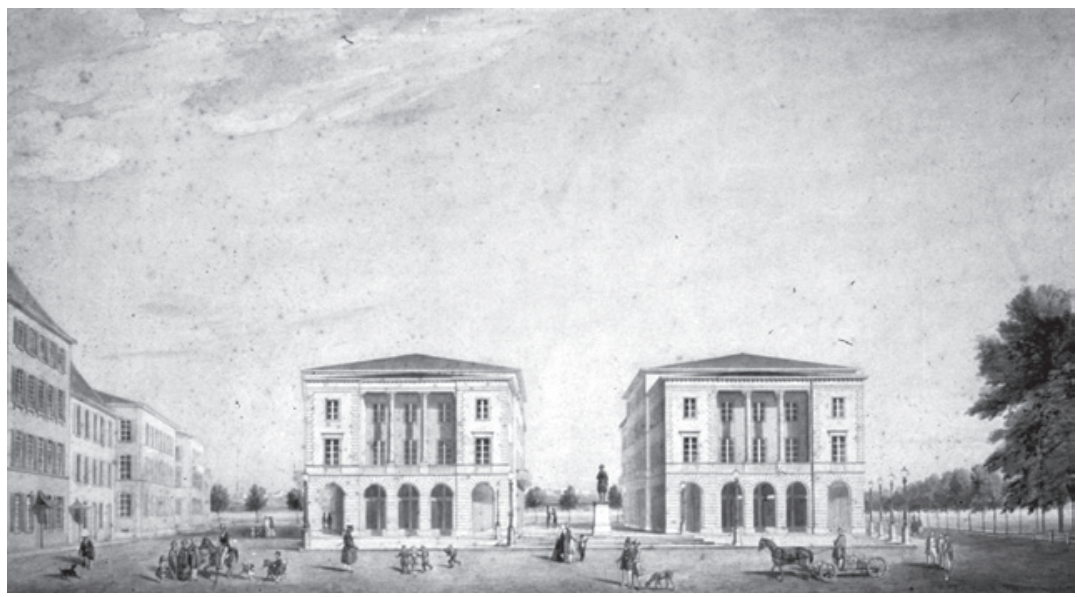

Fig. 7 - Façades monumentales du massif sud de la place Pury, aquarelle de H. Dietrich vers 1856. (Musée d'art et d'histoire, Neuchâtel).

de taille, un vocabulaire architectural et décoratif emprunté au classicisme, c'est un quartier recherché par une clientèle aisée.

Faute de documents de première main, le témoignage de l'ancien ingénieur des Ponts et Chaussées de la Principauté confirme qu'investir dans la Société de construction s'est révélé une bonne affaire financière pour les actionnaires, ainsi que pour les autorités qui auront en outre réussi à insuffler un élan important à la construction:

La Société de construction de Neuchâtel a été constituée pour le terme de vingt ans, en mai 1858, au capital de fr. 1000000 , qui a été entièrement versé ; elle est par conséquent entrée en liquidation en mai 1878. Pendant la période de son activité, elle a construit pour son compte vingt-neuf bâtiments qui lui ont coûté un peu plus de fr. 2000000 , en sorte que la valeur moyenne de chacun d'eux est de fr. 70000 . Elle a construit, pour le compte de tiers, sept bâtiments, entre autres les hôtels de la Banque cantonale, de la Caisse d'épargne et du Mont-Blanc, pour lesquels elle a perçu près de fr. 1800000 . Si, à ces opérations, on ajoute les achats et ventes de terrains, qui dépassent fr. 300000 , on trouve que, avec son capital de fr. 1000000 , cette société est parvenue à réaliser un mouvement d'affaires quadruple 
pendant une période de 20 ans, dont une grande partie a été peu favorable à ce genre de spéculations ${ }^{45}$.

\section{La répartition des rôles}

En l'absence d'archives, beaucoup de points restent à élucider quant aux critères et aux bases contractuelles qui lient architectes et sociétés de construction, ainsi que sur les conditions dans lesquelles les options architecturales sont déterminées.

En accord avec les statuts de la société qu'il dirige, le Conseil d'administration dispose de l'essentiel du pouvoir sous le contrôle de l'Assemblée générale qui le nomme, détermine les objectifs et approuve les comptes. A l'exception des exécutifs des sociétés de Vieux-Châtel et de construction, il ne comprend pas d'architecte ou d'ingénieur, même s'il fait office de commanditaire des travaux.

Défenseur des actionnaires et d'une certaine rentabilité, le Conseil d'administration choisit sans surprise une architecture sobre - en général néoclassique -, ainsi qu'une mise en ouvre traditionnelle et l'emploi de matériaux locaux. La standardisation des besoins conduit à des réalisations assez ordinaires, à l'exception de celles qui s'adressent à une clientèle précise ou qui remplissent une fonction spécifique comme l'hôtel Bellevue ou le massif sud de la place Pury.

Indépendant ou financièrement engagé, membre fondateur, petit ou gros actionnaire, le statut de l'architecte au sein de la société revêt à nouveau de multiples aspects, mais détermine la nature de sa participation (mandat, engagement, etc.). Son nom n'apparaissant qu'épisodiquement dans les documents publics, la figure du maître d'œuvre tend à s'effacer au profit de la société, même s'il amène sa maîtrise des programmes architecturaux, des techniques constructives et du suivi de chantier.

Au bénéfice d'une formation académique et membres de familles actives dans le bâtiment, Hans Rychner, Louis et Léo Châtelain, Guillaume Ritter et Gustave de Pury se distinguent des entrepreneurs par leur formation académique, mais ne contribuent que rarement à la monumentalisation des réalisations des sociétés pour lesquelles ils

45. G. de Pury, «L'activité neuchâteloise dans le domaine de la construction pendant les trente dernières années", p. 264. 
travaillent. A côté de l'édification de bâtiments publics, cette architecture alimentaire ne doit pas être négligée et constitue une facette peu documentée de l'activité de certains architectes neuchâtelois qui se profilent comme hommes d'affaires plutôt que comme artistes tels que le conçoit la formation des Beaux-Arts.

\section{Les actionnaires}

En 1865 Eugène Jeanjaquet, conseiller municipal, constate une certaine réserve des milieux financiers traditionnels:

Les grands essais se font en général par des personnes riches; or, les personnes riches de Neuchâtel, tout en acceptant très libéralement, je le reconnais, le nouvel ordre de choses, n'en sont pourtant pas encore venues à faire des sacrifices pour tenter d'y introduire l'industrie ${ }^{46}$.

Après les bouleversements politiques de 1848 et de 1856, il serait tentant d'opposer les membres de l'ancienne aristocratie à la classe émergente, les nouveaux venus éclairés et entreprenants butant sur la frilosité des milieux financiers conservateurs. Méfions-nous toutefois des généralisations hâtives. Un rapide survol de la composition de l'actionnariat des sociétés créées à Neuchâtel à la fin des années 1850 montre une situation beaucoup plus complexe et nuancée. Ces sociétés regroupent en général des personnalités domiciliées dans la région, mais provenant d'horizons variés: ingénieurs, architectes, rentiers, propriétaires, banquiers, notaires, avocats, professeurs, directeur des Postes, brasseur, négociants, etc. La fibre économique des patriciens neuchâtelois ne tarde pas à refaire surface et les exemples de membres de l'ancienne élite qui n'hésitent pas à investir dans l'industrie, les infrastructures, les nouvelles technologies et bien sûr l'immobilier ne font pas défaut, pas plus que les personnes nouvellement fortunées qui s'empressent de placer tout ou partie de leurs capitaux dans de la pierre ou des rentes foncières.

Une analyse fine permettrait probablement de montrer les regroupements par affinités et intérêt pour un projet, ainsi que par réseaux

46. E. Jeanjaquet, Agrandissement de Neuchâtel, continuation du quai du Gymnase, discours de M. Eugène Jeanjaquet en Conseil général de la Municipalité, le 2 juin 1865, p. 15. 
sociaux, professionnels et familiaux, sans oublier que le domaine immobilier attire facilement les maîtres d'état et les professionnels du bâtiment qui espèrent tirer profit de cet encouragement à la construction. Encore meurtries par les clivages politiques, les élites neuchâteloises ne sont pas complètement débarrassées des rancœurs et des réflexes partisans, mais elles se cherchent un nouvel équilibre. Aux côtés de ces investisseurs somme toute traditionnels, la surprise réside par contre dans la présence des... pouvoirs publics!

\section{Des autorités qui se font entrepreneur}

Confrontées à une situation économique peu brillante, les nouvelles autorités vont s'efforcer de susciter un élan économique ${ }^{47}$. Dès son entrée en fonction en 1856, la nouvelle Municipalité s'inscrit en rupture de l'administration précédente jugée attentiste et conservatrice et « doit se garder de suivre les anciens errements et faire sentir à ses administrés qu'elle est une institution jeune, pleine de sève et de vigueur. Comme Neuchâtel, depuis longtemps plongée dans une espèce de marasme, finirait par périr par consomption, il est urgent que le Conseil municipal avise aux moyens de le tirer de ce funeste état. Deux éléments sont nécessaires pour atteindre le but: l'industrie et le commerce " ${ }^{48}$, ainsi que leur corollaire: des locaux industriels et des logements.

Dans l'espoir d'«inaugurer sans plus tarder à Neuchâtel une ère d'amélioration et de progrès", la Municipalité dresse l'inventaire des travaux à entreprendre pour offrir des conditions de vie et de travail attrayantes : abattoirs, buanderies, chambre mortuaire, établissements de bains, port, gare, routes, pavages et trottoirs, éclairage au gaz, télégraphe, alimentation en eau, écoles primaires et industrielle, etc.

47. En comparaison de la bonne conjoncture économique qui prévaut autour d'elle, la ville de Neuchâtel connaît une certaine stagnation dont se plaignent les autorités. AVN, PV du Conseil municipal. E. Jeanjaquet, Agrandissement de Neuchâtel, continuation du quai du Gymnase, discours de M. Eugène Jeanjaquet en Conseil général de la Municipalité, le 2 juin 1865. S'il existe des études relatives à des secteurs ponctuels comme l'horlogerie, l'indiennerie ou la production de chocolat, une véritable analyse de l'économie neuchâteloise dans sa globalité manque pour la seconde moitié du $\mathrm{XIX}^{\mathrm{e}}$ siècle.

48. AVN, PV du Conseil municipal, 27 avril 1857. 
La Municipalité entend également encourager les milieux immobiliers et provoquer un mouvement en faveur de la construction de logements, en mettant près de 500000 francs à la disposition des sociétés de construction qui se constituent en 1858-1859, selon la clé de répartition suivante:

- Société du Manège: subvention de fr. 5000.-

- Société de l'Hôtel Bellevue: prise d'actions pour la valeur du terrain vendu

- Société immobilière pour la classe ouvrière: fr. --.-

- Société de Vieux-Châtel : prêt de fr. $150000 .-{ }^{49}$

- Société de construction: prise d'actions pour fr. 250000 .- et prêt de fr. $75000 .-{ }^{50}$

Mais pour moderniser la ville et la doter d'infrastructures et de logements, il faut avoir les moyens - notamment financiers - de ses ambitions.

\subsection{Se donner les moyens de ses ambitions}

En 1856-1857, l'équilibre entre spéculation et œuvre d'utilité publique, ainsi que les questions de gestion urbaine se posent déjà en termes étonnamment proches de ceux du XXI ${ }^{\mathrm{e}}$ siècle. Deux solutions se présentent à la Municipalité pour parvenir à ses fins: entreprendre et financer elle-même les projets d'utilité publique ou octroyer des concessions à des «capitalistes" privés qui assument les risques financiers. Ne disposant pas des moyens de la première approche et la seconde démarche ne rencontrant pas le succès escompté, les autorités doivent malheureusement constater que "l'industrie privée n'a pas encore, sous ce rapport, acquis chez nous le développement qu'elle a atteint dans d'autres villes, où depuis longtemps des spéculateurs se présentent à l'envi pour entreprendre les services publics mis en adjudication " ${ }^{51}$.

Pragmatique, la Municipalité imagine alors des solutions mixtes, comme la prise de participation dans des sociétés en commandite ou

49. AVN, Conventions, vol. a, n 5, convention entre la Municipalité et Maret Ritter et Cie, 18 septembre 1858 .

50. AVN, Conventions, vol. a, $n^{\circ} 3$ et $n^{\circ} 4$, conventions entre la Municipalité et la Société anonyme de construction de Neuchâtel, $1^{\text {er }}$ juillet 1858 et 17 septembre 1858 .

5I. AVN, Rapport de gestion pour l'exercice 1857, p. 7. 
anonyme (Fabrique des télégraphes et appareils électriques, usine à gaz et Société des eaux) ou le soutien à des associations privées par des prêts.

\subsection{D'où proviennent des moyens financiers d'une telle ampleur?}

Jusqu'au règlement des questions financières entre les administrations communale et municipale en 1861, la Municipalité peine à toucher sa part de la succession de David de Pury, un héritage qui assurait depuis 1786 à la Bourgeoisie de confortables revenus ${ }^{52}$. Dans de telles conditions, comment les autorités parviennent-elles à trouver les 500000 francs qu'elles mettent à disposition de sociétés susceptibles de construire des logements?

Dans le contexte de l'époque, lever un impôt municipal est non seulement considéré comme un "moyen extrême» ${ }^{53}$ et peu rémunérateur, mais également comme une mesure "dangereuse et impolitique" ${ }^{54}$. Au moment de son introduction en 1858, l'impôt rapporte annuellement 30000 à 40000 francs à la caisse municipale, une somme effectivement modeste au vu des besoins ${ }^{55}$.

Pour pallier rapidement à cette situation, la Municipalité décide d'emprunter un million de francs, une recherche de fonds qui n'est pourtant pas gagnée d'avance:

L'instant n'était pas favorable pour recourir au crédit public ; une crise financière sévissait, ébranlant le monde entier, l'argent était rare partout, et les caisses ne s'ouvraient guère que pour recevoir ; on ne prêtait plus ${ }^{56}$.

En octobre 1857, elle conclut néanmoins un emprunt à des conditions jugées intéressantes auprès des établissements Reinach et Oppenheim,

52. L.-E. Roulet (dir.), David de Pury 1709-1786.

53. AVN, Rapport de gestion pour l'exercice 1857, p. 20.

54. AEN, MCE, ratification d'un emprunt d'un million de francs par la Municipalité, 21 septembre 1857.

55. Fr. 32069 en 1858. Fr. 32837,90 en 1859. Fr. 41519,85. Si l'impôt récolté a peut-être permis d'assurer le service de la dette et de faciliter la mobilisation du capital, aucun document ne permet d'affirmer qu'il soit attribué à un poste spécifique. AVN, Rapport de gestion pour l'exercice 1858, p. 22; Rapport de gestion pour l'exercice 1859, p. 95 et Rapport de gestion pour l'exercice 1860, p. 24.

56. AVN, Rapport de gestion pour l'exercice 1857, p. 20. 
"maisons de banque de Bruxelles et Francfort-sur-Main " ${ }^{57}$. Avec des sommes "destinées à être placées [dans des sociétés] \& non point à être employées " ${ }^{58}$, les autorités font des placements profitables, tout en jouant un rôle actif: elles investissent dans des projets d'intérêt général et suscitent un nouvel élan à la croissance de Neuchâtel.

\section{Le client de l'architecte: une personnalité morale à multiples facettes}

Cette première étude consacrée aux sociétés immobilières en ville de Neuchâtel ne conduit pas à une image homogène. Les sociétés de construction poursuivent en effet une grande variété d'objectifs, qui se reflète dans leur organisation et leur envergure. En rupture avec l'héritage de l'Ancien régime, les sociétés immobilières inaugurent une nouvelle logique économique, produisant une architecture et un urbanisme qui cherche encore ses modes de fonctionnement et ses repères.

Plutôt que de parler d'un maître de l'ouvrage sans personnalité ou impersonnel, il faudrait plutôt parler d'une figure collective et d'une personnalité morale à lire en filigrane. Et cette lecture réserve parfois des surprises comme la discrétion de la figure de l'architecte et l'absence des milieux bancaires, au profit de la forte implication des autorités:

Si en 1858 nous avions écouté, et ils étaient nombreux, ceux qui nous disaient qu'une administration ne doit pas se faire entrepreneur, aurions-nous mis 400 mille francs à la disposition de deux sociétés de construction, et donné par là à cette capitale l'impulsion qu'elle a reçue et qu'il était temps enfin qu'elle reçut pour recouvrer un rang qu'elle n'aurait jamais dû perdre? Nous provoquâmes alors un grand mouvement dans la bâtisse ${ }^{59}$.

Claire Piguet

Office de la protection des monuments et des sites, Neuchâtel

57. AVN, Rapport de gestion pour l'exercice 1857, p. 21.

58. AVN, procès-verbaux du Conseil général, 14 juin 1858.

59. E. Jeanjaquet, Agrandissement de Neuchâtel, continuation du quai du Gymnase, discours de M. Eugène Jeanjaquet en Conseil général de la Municipalité, le 2 juin 1865, p. 15. 


\section{BIBLIOGRAPHIE}

\section{Sources}

Châtelain, Léo, "Rapport sur les habitations à bon marché», Bulletin de la Société neuchâteloise pour l'avancement des Sciences sociales, (1868), p. 91-125.

Jeanjaquet, Eugène, Agrandissement de Neuchâtel, continuation du quai du Gymnase, discours de M. Eugène Jeanjaquet en Conseil général de la Municipalité, le 2 juin 1865, Neuchâtel, Imprimerie Montandon frères, 1865.

Pury, Gustave de, "L'activité neuchâteloise dans le domaine de la construction pendant les trente dernières années, discours d'ouverture de la $28^{\mathrm{e}}$ assemblée générale de la Société suisse des Ingénieurs et Architectes, 18 août 1879", Musée Neuchâtelois, (1879), p. 231-242 et 247-265.

Quartier-la-Tente, Edouard, Le canton de Neuchâtel, le district de Neuchâtel, revue historique et monographique des communes du canton, le district de Neuchâtel, vol. 2, Neuchâtel, Attinger frères éditeurs, 1898.

\section{Etudes}

Bauer, Eddy, Autour d'une place, Neuchâtel, 1969.

Bauermeister, Nicole (dir.), Utopierre, Guillaume Ritter, de VieuxChâtel à Neuchâtel, Le Locle, Editions G d'encre, 2009.

Cotelli, Jean-Baptiste, "Les immeubles de Vieux-Châtel, un événement unique", in Utopierre, Guillaume Ritter, de Vieux-Châtel à Neuchâtel, dir. Nicole Bauermeister, Le Locle, Editions G d'encre, 2009, p. 61-87. 
Courvoisier, Jean, Girardbille, Olivier, Jelmini, Jean-Pierre et Rodeschini, Christine, "Neuchâtel, histoire d'un paysage urbain", Nouvelle revue neuchâteloise, 59 (numéro spécial, 1998), p. $1-59$.

Piguet, Claire, "Neuchâtel", in Inventaire suisse d'architecture 18501920, vol. 7, Berne, Société d'histoire de l'art en Suisse, 2000, p. 39-275.

—, "Mécanismes urbains et pièces d'architecture", in Le Pays de Neuchâtel et son patrimoine horloger, dir. Jacques Bujard et Laurent Tissot, Chézard-Saint-Martin, Editions de la Chatière, 2008, p. 181-199.

—, «Arrêt sur la ville: vivre et penser Neuchâtel en 1860 », Utopierre, Guillaume Ritter, de Vieux-Châtel à Neuchâtel, dir. Nicole Bauermeister, Le Locle, Editions G d'encre, 2009, p. 47-58.

—, «Panorama de la construction de logements à bon marché dans le canton de Neuchâtel (1850-194)", in Logement ouvrier, (Cahiers d'histoire du mouvement ouvrier 25), Lausanne, Editions d'En bas, 2009, p. 7-27.

—, "Guillaume Ritter: un arroseur arrosé?", in Neuchâtel, cité de l'eau, dir. Roland Stettler, Blaise Mulhauser, Blaise et Pierre-Olivier Aragno, Neuchâtel, Muséum d'histoire naturelle, 2009, p. 24.

Roulet, Louis-Edouard (dir.), David de Pury 1709-1786, Hauterive, Editions G. Attinger, 1986.

\section{Crédits photographiques}

Fig. 1, 5-7:

Office cantonal de la protection des monuments et des sites, Neuchâtel.

Fig. 3 et 4 :

Reno Sterchi. 